\title{
Effect of core training exercises on some physical and technical skill abilities in young soccer players
}

\section{DR . Mohamed Hamido Mahmoud}

\section{Introduction:}

The area named as Core is the area including the abdominals in the front side of the body, and Core exercises training refers to the training of the above mentioned abdominal and lumbar regions.

The area named as Core is the area including the abdominals in the front side of the body, and Core exercises training refers to the training of the above mentioned abdominal and lumbar regions.

In general, soccer is a team sport of intense tackles, and strong central body area decreases risks of injury and provides explosive power in soccer players, improved higher rate anaerobic energy, and technical skills with and without a ball.

There are many training methods for developing strength and power. With the development of these, skills such as vertical jump, speed, acceleration, agility, ball shooting, turning and dribbling can performed easily (Bangsbo, 1994; Bompa \& Haff, 2009; Cometti et al., 2001; Wisloeff et al., 1998).

In context of efficiency of core exercise in soccer players, (Borghuis et al., 2008) reported that, decreased core stability was reported to be associated with a higher risk of injuries of low back or knee and more improvement for physical fitness and technical skills.

Since the prevalence of knee injuries is very high in football, a widely practiced activity whether recreationally or competitively, the assessment of core stability in football players is of great importance (Nikolaidis, 2010). 
On the issue of which strength training method is more convenient for soccer training, primarily and certainly, it can be stated that the strength training methods do not depend only on the number of units per training done during soccer exercises (Weineck, 2011).

Core muscles aid in coordinating upper and lower body synchronous movement. This is important when soccer players moving with ball and even simply making the ball go where the player is intending.

Core stability and strength exercises are a common practice in soccer world, despite the lack of scientific support about their relation with performance. The purpose of this study was to examine the effect of core exercises on some physical and skill abilities of young soccer players. This is essential for jumping, kicking the ball, shooting accuracy, Juggling, and the explosive speed to compete for loose balls in the final minutes of games.

\section{Material and Methods:}

\section{Study design}

In the current study, pre-test and post-test with experimental method by using one experimental group identified for investigation all parameters in study.

\section{Subject}

Sixteen young soccer players from Smouha club in Alexandria city participated in current study. All Players registered in the Egyptian federation of soccer and competed in the Egyptian U16 league. The descriptive data of players in age, height, weight and training years were; $15.40 \pm 0.5$ years, $173 \pm 4.2 \mathrm{~cm}, 70.2 \pm 6.3 \mathrm{Kg}$ and $6.5 \pm 1.8$ years, respectively.

\section{Experimental procedures}

The experiment was carried out within a single testing session. Prior to each testing session, subjects completed a standard warm-up that consisted of a 5 to 10 minutes jog, a series of stretches, and a sequence of 20 meter running drills. Each skill test was separated by 
at least a 5 minutes recovery period, but players were allowed more rest if they so required and completed the series of tests in a similar order.

\section{Equipments}

- Restameter Pe 3000

- Tape measure in centimeters

- Medicine Ball $3 \mathrm{Kg}$

- Table

- Cones
- Medical scale for weight

- Tape marker

- Casio stopwatch

- Soccer balls

- Soccer field with goal target

\section{Measurements}

\section{Physical abilities tests}

The physical fitness tests battery that were used: vertical jump, standing long jump and medicine ball throw (explosive power); sprint 30 meter and shuttle run (speed and agility); and trunk flexor, trunk extensor and Lateral musculature (Core strength tests).

\section{(1) Vertical jump}

Vertical jump test was conducted with "Jump Meter". Players jumped upwards on a time and distance scaled sensitive surface in force without taking a step and skipping (Hoff, 2005). The distance they jumped was determined on the vehicle in centimeters. After players jumped twice, their best score was recorded as their vertical jump value.

\section{(2) Standing long jump}

A line was drawn on the field where the standing long jump test was done and from this line forward, a tape measure with a 0.01 sensitivity level was placed on the ground. In standing position, the players were placed in a way that their toe ends touch the line and were asked to jump forward. The players stopped at the place where their feet first touched the ground and the distance between the line and the players' heels was measured and recorded in centimeters. The measurement was done thrice and the best score was recorded (Vaeyens et al., 2006). 


\section{(3) Medicine Ball Throw}

This test evaluated the power of the upper body (Kukolj et al., 2003). The standing subjects were asked to throw the medicine ball with both hands as far as possible in the fashion of throwing in a real game. The distance was measured to the nearest $0.20 \mathrm{~m}$.

\section{(4) Sprint 30 meter}

The players ran on a $30 \mathrm{~m}$ field with maximal speed. The time was recorded in seconds with a Casio watch. The test was repeated twice on the participants and the best score was recorded (Hoff, 2005).

\section{(5) Agility shuttle run}

The shuttle run was used to determined agility performance. A distance of 10 yards was measured with a line at the 5 yard point. Participants straddled the middle line and ran to their left to the end of the 10 yard marker, then to their right to the opposite 10 yard marks, and back to the middle 5 yard point (Vaeyens et al., 2006). Time began with initial movement and ended when the individual crossed the 5 yard point a second time covering a total distance of 20 yards. Two timers were used with the average of the two recorded to the 0.01 second. Individuals were allowed three attempts with a 3-5 minutes rest between attempts. The best of the three attempts was used for data analysis.

\section{(6) Core Tests}

The protocol established by (Nikolaidis, 2010) was used to determine the core strength. The protocol consists of three tests that measure all aspects of core strength.

\section{Trunk flexor}

Trunk flexor test begins with the player in a sit-up position with the back resting against a jig angled at 60 degrees from the floor. Both knees and hips are flexed 90 degrees, the arms are folded across the chest with the hands placed on the opposite shoulder, and the feet are 
secured. To begin, the jig is pulled back $10 \mathrm{~cm}$ and the person holds the isometric posture as long as possible. Failure is determined when any part of the person's back touches the jig.

\section{Trunk extensor}

Trunk extensor test are tested with the upper body cantilevered out over the end of the test bench and with the pelvis, knees, and hips secured. The upper limbs are held across the chest with the hands resting on the opposite shoulders. Failure occurs when the upper body drops below the horizontal position.

\section{Lateral musculature}

Lateral musculature is tested with the person lying in the full side-bridge position (e.g., left and right side individually). Legs are extended, and the top foot is placed in front of the lower foot for support. Subjects support themselves on one elbow and on their feet while lifting their hips off the floor to create a straight line from head to toe. The uninvolved arm is held across the chest with the hand placed on the opposite shoulder. Failure occurs when the person loses the straight-back posture and/or the hip returns to the ground.

\section{(2) Soccer technical skills tests}

Four soccer-specific skills tests were used (Vaeyens et al., 2006). The slalom dribble test required players to navigate a ball around nine cones ( $2 \mathrm{~m}$ apart) from the start to end lines and return (better of two trials). The lob pass test required the player to kick a soccer ball from a distance of $20 \mathrm{~m}$ into an area divided into three concentric circles $(3,6$ and $9.15 \mathrm{~m}$ in diameter). Each kick was scored by the circle in which the ball initially landed (3, 2 and 1 points, respectively). Ten attempts (five with each foot) were allowed with a maximum of 30 points. Shooting accuracy test required the player to kick the ball at a $16 \mathrm{~m}$ wide goal target from a distance of $20 \mathrm{~m}$. The goal was divided into five parallel goals: centre, $2 \mathrm{~m}$ wide (3 points); two areas $3 \mathrm{~m}$ on each side of the center ( 2 points); and two areas $4 \mathrm{~m}$ wide at each extreme (1 point). Ten shots (five with each foot) were allowed with a maximum of 30 points. 
For the juggling test, the number of times players touched the ball before it bounced on the ground was recorded. The juggling test (two trials) had a maximum score of 200 points (100 per attempt). The soccer tests were performed on a soccer field and players wore soccer clothing and shoes.

\section{Core exercises training}

The core exercises training performed twice per week during normal training times and for 10 weeks in the intervention period, all players continued to perform their normal physical training, which consisted of skills training and run-based conditioning. The normal training program of young soccer players was three sessions per week and the session time is 120 minutes. Core exercises training took approximately 25 minutes to complete and was supervised by the principal investigator to ensure compliance and to maintain optimal exercise technique. For example, the core training period during the training session of young players included some exercises with swiss ball. Every player was provided with a swiss ball, sized such that, when they were seated on the vertical apex of the ball, the thighs were slightly above horizontal.

In addition, the core exercises consist of 4 different positions held for 30 second each. Exercise modification- If players cannot hold the position for the required time, they were instructed to hold the exercise as long as possible and then drop down to the floor to rest for 1 to 2 seconds and then reposition back to the exercise and continue with holding and resting throughout the 30 second interval. The exercises given were side plank, front plank position, twist curl exercise, supine bridging. For the modified plank position, the body remains straight and the position is held for 30 seconds. The following table demonstrates an overview of core exercises and skill drills, which implemented within research

Table 1. Example of core exercises and skill drills training implemented within study

\begin{tabular}{c|c|c|c|c}
\hline Exercises & $\mathbf{1 - 3}$ weeks & 4-6 weeks & $\mathbf{7 - 8}$ weeks & 9-10 weeks \\
\hline Swiss ball & 10 rep & 15 rep & 10 rep & 15 rep \\
\hline
\end{tabular}




\begin{tabular}{l|c|c|c|c}
\hline Side plank & $20 \mathrm{sec}$ & $25 \mathrm{sec}$ & $20 \mathrm{sec}$ & $25 \mathrm{sec}$ \\
\hline Front plank & $15 \mathrm{sec}$ & $20 \mathrm{sec}$ & $23 \mathrm{sec}$ & $25 \mathrm{sec}$ \\
\hline Twist medicine ball & $20 \mathrm{sec}$ & $25 \mathrm{sec}$ & $20 \mathrm{sec}$ & $25 \mathrm{sec}$ \\
\hline Jump & $15 \mathrm{rep}$ & $20 \mathrm{rep}$ & $15 \mathrm{rep}$ & $20 \mathrm{rep}$ \\
\hline Supine bridge & $25 \mathrm{sec}$ & $30 \mathrm{sec}$ & $25 \mathrm{sec}$ & $35 \mathrm{sec}$ \\
\hline Squat & $20 \mathrm{rep}$ & $25 \mathrm{rep}$ & $20 \mathrm{rep}$ & $25 \mathrm{rep}$ \\
\hline Lying twist trunk & $20 \mathrm{sec}$ & $25 \mathrm{sec}$ & $20 \mathrm{sec}$ & $25 \mathrm{sec}$ \\
\hline Dribbling & $5 \mathrm{rep}$ & $5 \mathrm{rep}$ & $4 \mathrm{rep}$ & $5 \mathrm{rep}$ \\
\hline Lobbing & $3 \mathrm{rep}$ & $5 \mathrm{rep}$ & $3 \mathrm{rep}$ & $5 \mathrm{rep}$ \\
\hline Shooting & $5 \mathrm{rep}$ & $5 \mathrm{rep}$ & $4 \mathrm{rep}$ & $5 \mathrm{rep}$ \\
\hline Juggling & $20 \mathrm{sec}$ & $25 \mathrm{sec}$ & $20 \mathrm{sec}$ & $25 \mathrm{sec}$ \\
\hline
\end{tabular}

\section{Statistical analysis}

Descriptive statistics were performed on all data. Independent t-test was computed to compare significant differences between pre and posttests in selected measurements and Pearson correlation coefficient test were used to identify relationships between test variables.

Statistical significance was set at $\mathrm{P} \leq 0.05$. SPSS 17.0 software was used for all analyses.

\section{Results:}

Results of the independent t-test are presented in table 2, and Pearson correlation coefficient test are presented in table 3 .

Table 2. Difference between pre-test and post-test in physical and skill abilities variables

\begin{tabular}{|c|c|c|c|c|c|}
\hline & Variables & Pre-test & Post-test & t-statistic & p-value \\
\hline \multirow{10}{*}{ 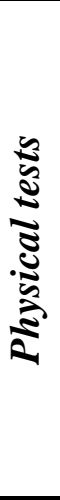 } & Vertical Jump (cm) & $28.64 \pm 2.96$ & $31.16 \pm 2.13$ & -12.51 & $0.000^{*}$ \\
\hline & Standing Long Jump $(\mathrm{cm})$ & $84.06 \pm 3.69$ & $104.76 \pm 5.79$ & -11.03 & $0.000 *$ \\
\hline & Medicine Ball Throw (m) & $7.80 \pm 2.19$ & $13.10 \pm 2.14$ & -11.28 & $0.000 *$ \\
\hline & Sprint 30 meter $(\mathrm{sec})$ & $4.25 \pm 0.20$ & $3.90 \pm 0.20$ & 2.63 & $0.000 *$ \\
\hline & Agility shuttle run (sec) & $20.90 \pm 1.10$ & $18.20 \pm 1.30$ & 6.40 & $0.000 *$ \\
\hline & Trunk flexor (sec) & $210 \pm 63.40$ & $290 \pm 34.20$ & 15.40 & $0.000 *$ \\
\hline & Trunk extensor $(\mathrm{sec})$ & $170 \pm 45.80$ & $225 \pm 52.50$ & 12.20 & $0.000 *$ \\
\hline & Lateral right musculature (sec) & $120.50 \pm 36.60$ & $210.90 \pm 22.30$ & 14.15 & $0.000 *$ \\
\hline & Lateral left musculature (sec) & $112.30 \pm 22.10$ & $198.70 \pm 26.40$ & 13.10 & $0.000 *$ \\
\hline & Total Core tests (sec) & $612.80 \pm 146.20$ & $924.60 \pm 152.80$ & 14.90 & $0.000 *$ \\
\hline \multirow{4}{*}{ 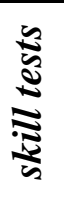 } & Slalom dribble (sec) & $17.40 \pm 1.10$ & $15.50 \pm 1.30$ & 6.80 & $0.000 *$ \\
\hline & Lob pass (points) & $21 \pm 4.60$ & $25.10 \pm 3.40$ & -10.64 & $0.000 *$ \\
\hline & Shooting accuracy (points) & $21.70 \pm 3.40$ & $25.80 \pm 2.70$ & -9.93 & $0.000 *$ \\
\hline & Juggling (nr) & $97.40 \pm 70.40$ & $117.70 \pm 53.30$ & -11.82 & $0.000 *$ \\
\hline
\end{tabular}


There are significant differences observed in table (2) between pre-test and post-test in all variables of physical and skills abilities variables.

Table 3. Correlations between total scores of core tests and physical and skill abilities

\begin{tabular}{|c|c|c|}
\hline \multicolumn{2}{|r|}{ Variables } & $(r)$ \\
\hline \multirow{5}{*}{ 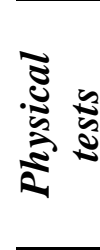 } & Vertical Jump (cm) & $0.732 *$ \\
\hline & Standing Long Jump (cm) & $0.870 *$ \\
\hline & Medicine Ball Throw (m) & $0.840 *$ \\
\hline & Sprint 30 meter $(\mathrm{sec})$ & -.362 \\
\hline & Agility shuttle run (sec) & -.476 \\
\hline \multirow{4}{*}{ 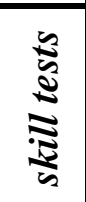 } & Slalom dribble (sec) & $0.756^{*}$ \\
\hline & Lob pass (points) & .391 \\
\hline & Shooting accuracy (points) & $0.845^{*}$ \\
\hline & Juggling (nr) & $0.821 *$ \\
\hline
\end{tabular}

There are significant correlations were identified in table (3) between total scores of core tests and physical and skill abilities, except the sprint over $30 \mathrm{~m}$, agility shuttle run and lob pass tests.

\section{Discussion:}

In general, the results of current study suggest that optimal core training is important for peak performance in soccer sport for young players. However, weak relationships observed between sprints, lob pass skill values with total scores of core tests. This study examined whether core exercises is good method for developing physical and technical skill abilities in young soccer players.

Overall, the results found that core training improve physical fitness abilities such as strength of upper and lower body, sprint and agility. In addition, core training improved specific soccer skills such as dribbling the ball and shooting accuracy. Compared with the pretest, the core exercises significantly increased vertical jump height, standing long jump and medicine ball throw $(8.8 \%, 24.6 \%$ and $67.9 \%)$, which was in agreement with the previous studies on youth soccer players using strength training methods (Gorostiaga et al., 2004; Moore et al., 2005). 
These results suggest that the core training is more important and positively affects the jump performance and contact body with opponents during play of young soccer players.

The core exercises significantly decreased the time of sprint over 30 meter and agility performance test $(8.2 \%$ and $14.8 \%)$, these results agreed with those in a previous study that reported a significant improvement in the $30 \mathrm{~m}$ sprint after a 13-week combined strength and speed training in youth soccer players (Kotzamanidis et al., 2005). Moreover, it has been reported that a short-distance sprint is highly dependent on the players' ability to generate muscular power (Delecluse et al., 1995), especially in the ankle, knee, and hip extensions (Frick et al., 1995).

Therefore, in the current study the core exercises training were improved sprint and agility performance because these exercises consisted of simultaneous triple-extension of the ankle, knee, and hip joints.

The core exercises significantly increased the total score time during core tests performance $(55.8 \%)$, which was in agreement with the previous studies using core training for improving balance and abdominal strength for soccer players (Hessari et al., 2011).

In context of soccer specific skills abilities, the core exercises significantly increased the ability of dribbling the ball, lop pass, ball-shooting accuracy and ball-juggling (10.9\%, 19.5\%, $18.9 \%$, and $20.8 \%$ ), which was in agreement with the previous study by (Kellis \& Katis, 2007; Manolopoulos et al., 2006) who reported that after a 10-week combined strength/plyometric and kicking training in amateur adult players, ball-shooting significantly increased and the strength of the vastus medialis of the swinging leg also increased significantly.

The core tests values in current study showed significantly correlated with all variables except the sprint parameters and lob pass skill test, which was in agreement with the previous study by (Kellis \& Katis, 2007) who reported a higher correlation between kicking 
performance and knee extension in high angular velocity $(r=0.90)$ among junior soccer players compared with that in low angular velocity $(\mathrm{r}=0.61)$.

Consequently, it was observed that 10 weeks core exercises combined with normal training implemented on young soccer players and provided improvements in physical and technical abilities. In addition, the core training plays an important part in the development of major and minor muscles; it will contribute to the motoric and physical developments of players positively.

\section{Conclusion:}

The importance of training for strong and stable core cannot be ignored. Core training that focuses on increasing the rate of strength and force development is essential, thus the core exercises that are functional and specific to young soccer players are important demands for improving performance in physical fitness and skills in soccer. In addition, the recommended measures of core area can be employed in any sport such soccer or exercise setting with minimum equipment, enabling soccer sport instructors and coaches to evaluate muscular strength and to determine optimal dosage of basic exertions such as sit-ups and back exercises.

\section{References:}

Bangsbo, Jens. (1994). Fitness training in football: a scientific approach: August Krogh Inst., University of Copenhagen.

Bompa, Tudor O.., \& Haff, Greg. (2009). Periodization: Theory and methodology of training: Human Kinetics.

Borghuis, Jan, Hof, At L, \& Lemmink, Koen APM. (2008). The importance of sensory-motor control in providing core stability. Sports medicine, 38(11), 893-916.

Cometti, G, Maffiuletti, NA, Pousson, M, Chatard, JC, \& Maffulli, N. (2001). Isokinetic strength and anaerobic power of elite, subelite and amateur French soccer players. International journal of sports medicine, 22(1), 45-51. 
Delecluse, C., Van Coppenolle, H., Willems, E., Van Leemputte, M., Diels, R., \& Goris, M. (1995). Influence of high-resistance and high-velocity training on sprint performance. Med Sci Sports Exerc, 27(8), 1203-1209.

Frick, U, Schmidtbleicher, D, \& Stutz, R. (1995). Muscle activation during accelerationphase in sprint running with special reference to starting posture. Paper presented at the XV Congress of the International Society of Biomechanics. Jyvaskyla, Finland.

Gorostiaga, E. M., Izquierdo, M., Ruesta, M., Iribarren, J., Gonzalez-Badillo, J. J., \& Ibanez, J. (2004). Strength training effects on physical performance and serum hormones in young soccer players. Eur J Appl Physiol, 91(5-6), 698-707.

Hessari, Famin Farzaneh, Norasteh, Ali Asghar, Daneshmandi, Hassan, \& Ortakand, Solmaz Mahdavi. (2011). The effect of 8 weeks core stabilization training program on balance in deaf students. Medicina Sportiva, 15(2), 56-61.

Hoff, Jan. (2005). Training and testing physical capacities for elite soccer players. Journal of sports sciences, 23(6), 573-582.

Kellis, E, \& Katis, A. (2007). The relationship between isokinetic knee extension and flexion strength with soccer kick kinematics: an electromyographic evaluation. Journal of Sports Medicine and Physical Fitness, 47(4), 385.

Kotzamanidis, C., Chatzopoulos, D., Michailidis, C., Papaiakovou, G., \& Patikas, D. (2005). The effect of a combined high-intensity strength and speed training program on the running and jumping ability of soccer players. J Strength Cond Res, 19(2), 369-375.

Kukolj, M, Ugarkovic, D, \& Jaric, S. (2003). Profiling anthropometric characteristics and functional performance of 12 to 18 -year-old elite junior soccer players. Journal of Human Movement Studies, 45(5), 403-418.

Manolopoulos, E., Papadopoulos, C., \& Kellis, E. (2006). Effects of combined strength and kick coordination training on soccer kick biomechanics in amateur players. Scand $J$ Med Sci Sports, 16(2), 102-110.

Moore, E. W., Hickey, M. S., \& Reiser, R. F. (2005). Comparison of two twelve week offseason combined training programs on entry level collegiate soccer players' performance. J Strength Cond Res, 19(4), 791-798.

Nikolaidis, Pantelis. (2010). Core stability of male and female football players. Biomedical Human Kinetics, 2, 30-33.

Vaeyens, Roel, Malina, Robert M, Janssens, Melissa, Van Renterghem, Bart, Bourgois, Jan, Vrijens, Jacques, \& Philippaerts, Renaat M. (2006). A multidisciplinary selection 
model for youth soccer: the Ghent Youth Soccer Project. British journal of sports medicine, 40(11), 928-934.

Weineck, J. (2011). Futbolda Kondisyon Antrenmani. Çev: T. Bağirgan). Spor Yayin evi ve Kitap evi. Spor kurami, 5, 194-195.

Wisloeff, ULRIK, Helgerud, J, \& Hoff, J. (1998). Strength and endurance of elite soccer players. Medicine and science in sports and exercise, 30, 462-467. 